\title{
THE EXISTENCE OF GENERALIZED ISOTHERMAL COORDINATES FOR HIGHER DIMENSIONAL RIEMANNIAN MANIFOLDS
}

\author{
JIANGUO CAO
}

\begin{abstract}
We shall show that, for any given point $p$ on a Riemannian manifold $\left(M, g^{0}\right)$, there is a pointwise conformal metric $g=\Phi g^{0}$ in which the $g$-geodesic sphere centered at $p$ with radius $r$ has constant mean curvature $1 / r$ for all sufficiently small $r$. Furthermore, the exponential map of $g$ at $p$ is a measure preserving map in a small ball around $p$.
\end{abstract}

\section{INTRODUCTION}

This note is devoted to studying the mean curvature of the geodesic sphere. In particular, we are interested in the existence of geodesic spheres with constant mean curvature under a certain conformal deformation of metrics.

In what follows, we always assume that $M^{n}$ is an $n$-dimensional $C^{\infty}$ smooth manifold and $g^{0}$ is an arbitrary Riemannian metric on $M$, simply denoted by $\left(M^{n}, g^{0}\right)$, and we also assume that $\Phi$ is a strictly positive function.

The goal of this paper is to prove the following.

Main Theorem. For any given point $p$ on a given $C^{\infty}\left(C^{k, \alpha}\right.$ resp.) Riemannian manifold $\left(M, g^{0}\right)$, there is a local $C^{\infty}\left(C^{k-3, \alpha}\right.$ resp.) pointwise conformal metric $g=\Phi g^{0}$ such that the geodesic sphere centered at $p$ with radius $\tilde{r}$ in the metric $g$ has constant mean curvature exactly $1 / \tilde{r}$ for all sufficiently small $\tilde{r}$.

To illustrate the usefulness of the main theorem, we present some applications.

First, the exponential map of the metric $g$ given in the main theorem has some nice properties. Let $\left\{y_{1}, \ldots, y_{n}\right\}$ be $g$-geodesic normal coordinates at $p$ and let $\operatorname{det}\left(g_{i j}(y)\right)$ be the determinant of the metric $g$ with respect to $\left\{y_{i}\right\}$. Using the main theorem, one can prove the following corollary (cf. §1).

Corollary 0.1. Let $\left(M, g^{0}\right)$ be a $C^{\infty}$ Riemannian manifold and let $p$ be a point on $M$. Then there exists a conformal metric $g=\Phi g^{0}$ such that $\operatorname{det} g_{i j}(y)=1$

Received by the editors January 10, 1989 and, in revised form, April 12, 1989.

1980 Mathematics Subject Classification (1985 Revision). Primary 53A10, 35J60; Secondary 53A30, 58G30. 
for all sufficiently small $\|y\|$, i.e., the exponential map of $g$ at $p, \operatorname{Exp}_{p}$, is a local volume preserving map in a small neighborhood of $p$.

Corollary 0.1 can be used to estimate the best constant in the critical Sobolev inequality which is related to the Yamabe problem (see [LP]). In order to simplify the proof of the Yamabe conjecture, using Graham's technique, Lee and Parker constructed a conformal metric $g$ with

$$
\operatorname{det} g_{i j}(y)=1+o\left(|y|^{N}\right),
$$

where $N$ is an arbitrary larger number. Lee and Parker were interested in knowing if det $g_{i j}(y)=1$ could be achieved exactly by some conformal normal coordinates in a small neighborhood of $p$. Now, this problem is solved by Corollary 0.1 .

For a 2-dimensional Riemannian surface $\left(M^{2}, g\right)$, one can show that $g$ is flat if and only if the mean curvature of a geodesic circle of radius $\tilde{r}$ is exactly equal to $1 / \tilde{r}$ for all sufficiently small $\tilde{r}$. Therefore, we can prove the main theorem for $n=2$ by using the classical isothermal coordinates of the metric $g^{0}$. A simple proof of the existence of the isothermal coordinates can be found in [DK]. For the higher dimensional Riemannian manifold $\left(M^{n}, g^{0}\right)$, the geodesic normal coordinates of the metric $g$ given in the main theorem give a natural generalization of the isothermal coordinates for $n \geq 3$.

Our proof of the main theorem introduces some new ideas. One of them is to use the singular elliptic theory to solve the geometric problem mentioned above. A similar singular elliptic problem was treated by C. Caffarelli, R. Hardt, and L. Simon in their joint paper [CHS]. (This was pointed out to the author by Professor Fanghua Lin.) However, our singular elliptic problem is more complicated than theirs. New prior estimates and a different argument are needed for the proof of the main theorem.

The plan of this paper is as follows: In $\S 1$, the singular elliptic equation corresponding to our geometric problem will be derived. We will obtain some prior estimates and solve the corresponding singular linear problem in $\S 2$. Using these estimates and the Schauder fixed point theory, we will prove the main theorem for some special metrics in $\S 3$. We shall also show that any $C^{\infty}$ smooth metric $g^{0}$ is conformal to one of those special metrics. Thus, the proof of the main theorem will be completed.

Conventions. All the manifolds are assumed to be $C^{\infty}$ and $g^{0}$ denotes a given $C^{\infty}$ smooth metric unless specified otherwise. $B_{r}(p)$ will stand for the metric ball of radius $r$ centered at $p$ and $r(x)$ is the distance function of $p$ with respect to the metric $g^{0}$. \|\|$_{g^{0}}$ will represented the norm in the metric $g^{0}$. $C^{k, \alpha}\left(B_{r}\right)$ is the usual Hölder space, i.e., the space of all functions whose first $k$ derivatives are continuous and the $k$ derivatives satisfy a Hölder condition with exponent $\alpha$ defined on the ball $B_{r}$. Let $E^{n}$ be the Euclidean space of dimension $n$. Our Laplacian, $\Delta$, has the sign such that $\Delta u=u^{\prime \prime}$ on $E^{1}$. 
We will let $\left\{x_{i}\right\}$ be $g^{0}$-geodesic normal coordinates about $p$ and $\operatorname{det} g_{i j}^{0}(x)$ be the determinant of $g^{0}$ with respect to the coordinates $\left\{x_{i}\right\}$. Similarly, if $g=\Phi g^{0}$, we define $\left\{y_{i}\right\}$ to be $g$-normal coordinates of $p$ and $\operatorname{det} g_{i j}(y)$ to be the determinant of $g$ with respect to $\left\{y_{i}\right\}$. These notations will be used throughout this paper.

\section{THE DISTANCE FUNCTION AND SINGULAR EQUATIONS}

In this section, we will use two basic facts about distance functions to derive a differential equation for our geometric problem.

First, let $p,\left(M, g^{0}\right)$, and \|\|$_{g^{0}}$ be as in the Introduction. Suppose $g=$ $\Phi g^{0}$ is a pointwise conformal metric of $g^{0}$ and $\tilde{r}(x)=d_{g}(x, p)$ is a distance function of a given point $p$ with respect to the metric $g$. Now, one observes that

where

$$
1=\|d \tilde{r}\|_{g}^{2}=\|d \tilde{r}\|_{\Phi g^{0}}^{2}=\frac{1}{\Phi}\|d \tilde{r}\|_{g^{0}}^{2}
$$

$$
\Phi=\|d \tilde{r}\|_{g^{0}}^{2}
$$

and

$$
g=\|d \tilde{r}\|_{g^{0}}^{2} g^{0} .
$$

Second, the distance function is related to the mean curvature of geodesic spheres as follows. Let $H_{\tilde{r}}(x)$ be the mean curvature of a $g$-geodesics sphere, centered at $p$ with radius $\tilde{r}$, at $x$. A direct computation yields that

$$
H_{\tilde{r}}(y)=\frac{1}{n-1} \Delta_{g} \tilde{r}
$$

(see [EH, p. 142]), where $\Delta_{g}$ is the Laplacian of the metric $g$ which has the sign such that $\Delta u=+u^{\prime \prime}$ on $E^{1}$.

Therefore, we conclude that

Theorem 1.4. Suppose $\left(M, g^{0}\right)$ is a $C^{\infty}$ ( $C^{k, \alpha}$ resp.) Riemannian manifold. Then the main theorem holds if and only if the equation

$$
\Delta_{\left(\|d \tilde{r}\|_{g^{0}}^{2}\right) g^{0}} \tilde{r}-\frac{n-1}{\tilde{r}}=0
$$

has a solution such that

$$
\Phi=\|d \tilde{r}\|_{g^{0}}^{2} \text { is a positive } C^{\infty}\left(C^{k, \alpha} \text { resp. }\right) \text { function, }
$$

$\tilde{r}$ is the distance function of given point $p$ with respect to the metric $g=\|d \tilde{r}\|_{g^{0}}^{2} g^{0}$.

Proof. Using (1.3), we know that the condition $H_{\tilde{r}}(x)=1 / \tilde{r}$ is equivalent to the condition

$$
\Delta_{g} \tilde{r}=(n-1) / \tilde{r}
$$


Combining (1.1), (1.2), and (1.7), we get equation $(*)$. Hence, Theorem 1.4 follows.

One should be aware that an arbitrary function is not necessarily a distance function. For example, the distance function has to be nonnegative. One should also notice that the distance functions are not necessarily smooth. For instance, in the Euclidean space $E^{2}, r(x)=\sqrt{x_{1}^{2}+x_{2}^{2}}$ is not $C^{1, \alpha}$ smooth at $x=0$. Therefore, we have to solve $(*)$ under several restrictions and one needs to find a criterion for distance functions.

Theorem 1.8. Suppose $U$ is a neighborhood of $p$ on a Riemannian manifold $(M, g), u^{2}$ is a $C^{3, \alpha}$ function defined on $U$, and we assume the following conditions hold:

(1) $u(p)=0$,

(2) $p$ is a critical point of $u^{2}$ and the Hessian of $u^{2}$ at $p$ is positive definite, and

(3) $\|d u\|_{g}=1$ holds $U-\{p\}$.

Then, there is an open set $B_{\varepsilon_{0}} \subset U$ such that $p \in B_{\varepsilon_{0}}$ and $u(x)=d_{g}(x, p)$ for all $x \in B_{\varepsilon_{0}}$.

Proof. Since $p$ is a critical point of $u^{2}$ and the Hessian of $u^{2}$ is positive definite at $p$, it follows from a lemma of Morse (see [M, p. 6]) that there is a local coordinate chart $\left\{z_{1}, z_{2}, \ldots, z_{n}\right\}$ such that $Z(p)=0, Z=\left(z_{1}, \ldots, z_{n}\right)$, and $u(Z)=z_{1}^{2}+\cdots+z_{n}^{2}$ if $\sum z_{i}^{2} \leq \varepsilon_{0}^{2}$. Setting

$$
\begin{aligned}
& B_{\varepsilon}=\left\{Z \mid \sum z_{i}^{2} \leq \varepsilon^{2}\right\}=\{Z \mid u(Z) \leq \varepsilon\}, \\
& S_{\varepsilon}=\left\{Z \mid \sum z_{i}^{2}=\varepsilon^{2}\right\}=\{Z \mid u(Z)=\varepsilon\},
\end{aligned}
$$

We will prove the following assertions:

Claim 1. There is a constant number $\delta>0$ such that

$$
\max _{Z \in S_{\varepsilon}} d_{g}(0, Z) \leq \delta \varepsilon \quad \text { for all } \varepsilon<\varepsilon_{0} .
$$

Claim 2. $d_{g}\left(S_{\varepsilon}, y\right)=u(y)-\varepsilon$ for all $\varepsilon<u(y)$.

Assuming Claims 1 and 2, we see that $d_{g}(0, y)=\lim _{\varepsilon \rightarrow 0} d_{g}\left(S_{\varepsilon}, y\right)=u(y)$. Hence, Theorem 1.8 follows immediately.

Claim 1 can be proved as follows: We let $g_{z_{i} z_{j}}=\left\langle\partial / \partial z_{i}, \partial / \partial z_{j}\right\rangle$ and

$$
\delta^{2}=\max _{Z \in \bar{B}_{\varepsilon_{0}}}\left\{\sum g_{z_{i} z_{j}}(Z) \zeta_{i} \zeta_{j} \mid \zeta_{1}^{2}+\cdots+\zeta_{n}^{2}=1\right\}
$$

For any $Z \in S_{\varepsilon}$, we take a curve $r_{z}: r_{z}(t)=\left(t z_{1}, \ldots, t z_{n}\right), t \in[0,1]$. The 
length of $r_{z}$ is computed as follows:

$$
\begin{aligned}
L\left(r_{z}\right) & =\int_{0}^{1}\left[\sum g_{z_{i} z_{j}}(t z) z_{i} z_{j}\right]^{1 / 2} d t \\
& \leq \int_{0}^{1} \delta \varepsilon d t=\delta \varepsilon .
\end{aligned}
$$

Therefore, $d_{g}(0, Z) \leq \delta \varepsilon$ for all $Z \in S_{\varepsilon}$. This finishes the proof of Claim 1 . Using the gradient flow of $(-u)$ on $B_{\varepsilon_{0}}-\{0\}$, one can prove Claim 2 (see [EH, p. 142]).

Using Theorem 1.8, we can construct a lot of distance functions as follows: Corollary 1.9. Let $p \in\left(M, g^{0}\right)$ be as before and let $r(x)=d_{g^{0}}(x, p)$ be the distance function of the given point $p$ with respect to the metric $g^{0}$. For any $C^{\infty}\left(C^{k, \alpha}\right.$ resp., $\left.k \geq 3\right)$ smooth function $w$, setting

$$
\tilde{r}(x)=r(x) e^{w(x)},
$$

we have

$$
\tilde{r}(x)=d_{g}(x, p) \text { when } g=\|d \tilde{r}\|_{g^{0}}^{2} g^{0},
$$

$$
\begin{aligned}
& \Phi=\|d \tilde{r}\|_{g^{0}}^{2} \text { is a positive } C^{\infty}\left(C^{k-1, \alpha} \text { resp. }\right) \text { smooth function } \\
& \text { for sufficiently small }|x| .
\end{aligned}
$$

Proof. Taking $g^{0}$-geodesic normal coordinates at $p$, one sees that $r(x)=$ $\left[\sum x_{i}^{2}\right]^{1 / 2}$ and $\tilde{r}^{2}=r^{2} e^{2 w}$ is a $C^{\infty}\left(C^{k, \alpha}\right.$ resp. $)$ smooth function. Furthermore,

$$
\|d \tilde{r}\|_{g^{0}}^{2}=\left(1+2 \sum_{i=1}^{n} x_{i} \frac{\partial w}{\partial x_{i}}+r^{2}\|d w\|_{g^{0}}^{2}\right) e^{2 w}
$$

is also a positive $C^{\infty}\left(C^{k-1, \alpha}\right.$ resp.) smooth function for sufficiently small $|x|$. Clearly, $\tilde{r}$ and $g=\|d \tilde{r}\|_{g^{0}}^{2} g^{0}$ satisfy the conditions of Theorem 1.8. Corollary 1.9 follows immediately.

Setting $\tilde{r}(x)=r e^{w(x)}$, we want to get an equation of $w(x)$ from $(*)$. With notations defined in the Introduction, it is easy to verify that

$$
\begin{aligned}
\Delta_{\Phi g^{0}} \tilde{r} & =\frac{1}{\Phi}\left\{\Delta_{g^{0}} \tilde{r}+\frac{n-2}{2}\langle d(\log \Phi), d \tilde{r}\rangle_{g^{0}}\right\} \\
& =\frac{1}{\Phi}\left\{\Delta_{g^{0}} \tilde{r}+\frac{n-2}{2}\left\langle d\left(\log \Phi_{1}\right), d \tilde{r}\right\rangle_{g^{0}}+(n-2)\langle d w, d \tilde{r}\rangle_{g^{0}}\right\},
\end{aligned}
$$

where

$$
\Phi_{1}=\left(1+2 \sum_{i=1}^{n} x_{i} \frac{\partial w}{\partial x_{i}}+r^{2}\|d w\|_{g^{0}}^{2}\right)=\Phi e^{-2 w}
$$


and

$$
\begin{aligned}
& \begin{aligned}
& \Delta_{g^{0}} \tilde{r}=\Delta_{g^{0}}\left(r e^{w}\right) \\
&=e^{w}\left[r\left(\Delta_{g^{0}} w+\|d w\|_{g^{0}}^{2}\right)+2 \frac{\partial w}{\partial r}+\Delta_{g^{0}} r\right], \\
& \begin{aligned}
\Delta_{g^{0}} r & =\frac{n-1}{r}+\frac{\partial}{\partial r}\left[\log \sqrt{\operatorname{det}\left(g_{i j}^{0}\right)}\right], \\
\left\langle d\left(\log \Phi_{1}\right), d \tilde{r}\right\rangle_{g^{0}} & =\left\langle d\left(\log \Phi_{1}\right), e^{2}(d r+r d w)\right\rangle \\
& =e^{w}\left[r\left\langle d\left(\log \Phi_{1}\right), d w\right\rangle+\frac{\partial}{\partial r}\left(\log \Phi_{1}\right)\right],
\end{aligned} \\
&\langle d \tilde{r}, d w\rangle=e^{w}\left[\frac{\partial w}{\partial r}+r\|d w\|_{g^{0}}^{2}\right] .
\end{aligned}
\end{aligned}
$$

Finally, we can write the equation of $w$ as

$$
\begin{aligned}
& 0=\Delta_{\left(\|d \tilde{r}\|_{g^{0}}^{2}\right) g^{0}} \tilde{r}-\frac{n-1}{\tilde{r}} \\
& =\frac{r}{\Phi_{1} e^{w}}\left\{\Delta_{g^{0}}(w)+\frac{n-2}{\Phi_{1}}\left[\frac{1}{2}\left\langle d \Phi_{1}, d w\right\rangle_{g^{0}}+\frac{\partial^{2} w}{\partial r^{2}}\right.\right. \\
& +\frac{1}{2 r} \frac{\partial}{\partial r}\left(r^{2}\|d w\|_{g^{0}}^{2}\right) \\
& \left.-\left(2 \frac{\partial w}{\partial r}+r\|d w\|_{g^{0}}^{2}\right) \frac{\partial w}{\partial r}\right] \\
& \left.+\frac{1}{r} \frac{\partial}{\partial r}\left(\log \sqrt{\operatorname{det}\left(g_{i j}^{0}(x)\right)}\right)\right\} \\
& =\frac{r}{\Phi_{1} e^{w}}\left\{V\left(x, \partial w, \partial^{2} w\right)+\frac{\partial_{r}\left(\log \sqrt{\operatorname{det}\left(g_{i j}^{0}\right)}\right)}{r}\right\},
\end{aligned}
$$

where $V$ is defined as

(1.14)

$$
\begin{aligned}
V\left(x, \partial w, \partial^{2} w\right) & \\
=\Delta_{g^{0}}(w)+\frac{n-2}{\Phi 1}[ & \frac{1}{2}\left\langle d \Phi_{1}, d w\right\rangle_{g^{0}}+\frac{\partial^{2} w}{\partial r^{2}} \\
& \left.+\frac{1}{2 r} \frac{\partial}{\partial r}\left(r^{2}\|d w\|_{g^{0}}^{2}\right)-\left(\frac{\partial w}{\partial r}+r\|d w\|_{g^{0}}^{2}\right) \frac{\partial w}{\partial r}\right] .
\end{aligned}
$$

Hence, Theorem 1.4 and Corollary 1.9 imply

Theorem A. Let $\left(M, g^{0}\right)$ be a $C^{k, \alpha}\left(C^{\infty}\right.$ resp. $)$ Riemannian manifold and let $V$ be as above. If $k \geq 5$ and the equation

$$
V\left(x, \partial w, \partial^{2} w\right)=-\frac{\partial_{r}\left(\log \sqrt{\operatorname{det}\left(g_{i j}^{0}(x)\right)}\right)}{r}
$$


has a $C^{k-2, \alpha}-\left(C^{\infty}\right.$-resp. $)$ solution in a neighborhood of $p$, then the main theorem holds.

Assuming that the main theorem holds, we would like to prove

Corollary 0.1. Let $\left(M, g^{0}\right)$ be a $C^{\infty}$ Riemannian manifold and let $p$ be a point on $M$. Then there exists a conformal metric $g=\Phi g^{0}$ such that $\operatorname{det}\left(g_{i, j}(y)\right)=1$ for all sufficiently small $|y|=\tilde{r}$, i.e., the exponential map of the metric $g$ at $p$ is a measure preserving map in a small neighborhood of $p$.

Proof. Let $g$ be given by the main theorem and $\left\{y_{i}\right\}$ be $g$-geodesic normal coordinates at $p$. One can verify

$$
\Delta_{g} \tilde{r}=\frac{n-1}{\tilde{r}}+\frac{\partial}{\partial \tilde{r}}\left[\log \sqrt{\operatorname{det}\left(g_{i j}(y)\right)}\right] .
$$

Using the fact that $\Delta_{g} \tilde{r}=(n-1) H_{\tilde{r}}=(n-1) / \tilde{r}$ and $(1.15)$, one gets

$$
\frac{\partial}{\partial \tilde{r}}\left[\log \sqrt{\operatorname{det}\left(g_{i j}(y)\right)}\right]=0 .
$$

But $\left.\operatorname{det}\left(g_{i j}\right)\right|_{y=0}=1$ for any Riemannian metric $g$. By solving the ordinary differential equation $(\partial / \partial \tilde{r})\left(\log \sqrt{\operatorname{det}\left(g_{i j}\right)}\right)=0$ along each radial direction $\partial / \partial \tilde{r}$ with the zero initial condition, we conclude that Corollary 0.1 is true.

In order to solve $(* *)$ we have to solve the corresponding linear equation in the next section.

\section{SOLUTIONS OF THE INHOMOGENEOUS LINEAR PROBLEM}

In $\S 1$, we found the differential operator $V$ given by (1.14) which is nonlinear. In this section, we will linearize the operator $V$ and solve the corresponding inhomogeneous linear problem.

The usual linearization of $V$ is too complicated to discuss in detail in this paper. Instead, we introduce the "scaling linearization" of $V$ as follows:

Let $\left\{x_{i}\right\}$ be $g^{0}$-geodesic normal coordinates at $p$; it is well-known that $g_{i j}^{0}(0)=\delta_{i j}$ and $\partial g_{i j}^{0}(0) / \partial x_{k}=0$. For any given function $v$, we set $w_{\rho}(x)=$ $\rho^{2} v(x / \rho), x=\rho y, r=\rho R$, and $R=\left[\sum y_{i}^{2}\right]^{1 / 2}=|y|$. A simple calculation shows that

$$
\begin{array}{rl}
\lim _{\rho \rightarrow 0} & V\left(x, w_{\rho}, \partial_{x} w_{\rho}, \partial_{x}^{2} w_{\rho}\right) \\
& =\lim _{\rho \rightarrow 0} V\left(\rho y, \rho^{2} v, \rho \partial_{y} v, \partial_{y}^{2} v\right) \\
& =\Delta_{E^{n}} v+(n-2) \frac{\partial^{2} v}{\partial R^{2}}
\end{array}
$$

where $\Delta_{E^{n}} v=\sum \partial^{2} v / \partial y_{i}^{2}$.

Naturally, we call the linear operator

$$
\mathscr{L}_{0}=\Delta_{E^{n}}+(n-2) \frac{\partial^{2}}{\partial R^{2}}
$$

the scaling linearization of $V$. 
The rest of this section is devoted to solving the inhomogeneous linear equation

$$
\mathscr{L}_{0} v=f
$$

where $\mathscr{L}_{0}$ is given by $(2.0)$.

We would like to make two comments about the inhomogeneous equation $\mathscr{L}_{0} v=f$. First, the usual regularity theory cannot be applied to this equation directly. For example, in the 3-dimensional Euclidean space $E^{3}, v=$ $|y|^{\sqrt{3}-2}\left(3 y_{1}^{2}-3 y_{2}^{2}\right)$ is a solution of $\mathscr{L}_{0} v=0$. Although $f=0$ is a smooth function, the solution $v$ is at most $C^{3}$. Secondly, for many $C^{\infty}$ smooth functions $v, \mathscr{L}_{0} v$ is not necessarily well defined at $y=0$. For instance, one can simply pick a linear function, say, $v=y_{1}$, then $\mathscr{L}_{0} v$ does not make sense at $y=0$.

These two examples remind us that we have to find the function subspaces on which $\mathscr{L}_{0}$ is well defined. The rapidly decaying function spaces with weighted Hölder norms are good choices for our $\mathscr{L}_{0}$. Let $B_{\rho}$ and $C^{k, \alpha}\left(B_{\rho}\right)$ be as in the Introduction. We define the weighted Hölder norms and spaces as follows:

$$
\||v|\|_{k, \alpha ; N, \rho}=\sup _{0<R<\rho}\left\{R^{-N}\left(|v|_{C^{k, \alpha}\left(B_{R}-B_{r / 2}\right)}\right\},\right.
$$

where $0<\alpha<1, k \leq N$, and

$$
\begin{aligned}
|v|_{C^{k, \alpha}\left(B_{R}-B_{R / 2}\right)} & \sum_{|j|=0}^{k} R^{|j|} \sup _{R / 2<|y| \leq R}\left\{\left|\partial^{j} v(y)\right|\right\} \\
& +R^{k+\alpha} \sup \left\{\frac{\left|\partial^{k} v(x)-\partial^{k} v(y)\right|^{\alpha}}{|x-y|^{\alpha}}|x \neq y, R / 2<| x|,| y \mid<R\right\},
\end{aligned}
$$

$$
C_{k, \alpha ; N, \rho}=\left\{v\left|v \in C^{k, \alpha}\left(B_{\rho}\right),\||v|\|_{k, \alpha ; N, \rho}<+\infty\right\}\right. \text {, }
$$

$$
Z_{\rho}=\bigcap_{k, N}^{\infty} C_{k, \alpha ; N, \rho}
$$

Clearly, $\mathscr{L}_{0}$ maps $C_{k, \alpha ; N, \rho}$ to $C_{k-2, \alpha ; N-2, \rho}$ and maps $Z_{\rho}$ to $Z_{\rho}$. In what follows, we are working on $C_{k, \alpha ; N, \rho}$ and $Z_{\rho}$. For simplicity, when $\rho=1$, we set

$$
C_{k, \alpha ; N}=C_{k, \alpha ; N, 1} \text { and }\|||\|_{k, \alpha ; N}=\|||\|_{k, \alpha ; N, 1} .
$$

In order to get the estimates of the singular equation (2.1), we need to define a weighted $L^{2}$ norm:

$$
i v i_{L_{N, \sigma}^{2}}=\sup _{0 \leq r \leq \sigma}\left\{r^{-N}\left[\int_{1 / 4 \sigma}^{1 / \sigma} \int_{S^{n-1}} t^{n-1} v^{2}(r t, \theta) d \theta d t\right]^{1 / 2}\right\} .
$$

Now, we are going to prove 
Lemma 2.7. If $a>0, b \geq 0$, and $1>\sigma>1 / 2$, then, for any weak solution of

$$
\mathscr{L}_{0} v=\left(a \Delta_{E^{n}}+b \frac{\partial}{\partial R^{2}}+\frac{c}{R} \frac{\partial}{\partial R}+\frac{d}{R^{2}}\right) v=f
$$

there exists a constant $K_{1}$ which is independent of $f$ and $v$ such that

$$
\||v|\|_{k, \alpha ; N, \sigma} \leq K_{1}\left\{\||f|\|_{k-2, \alpha, N-2}+v_{L_{N, \sigma}^{2}}\right\},
$$

where $a, b, \sigma$, and $c$ are constant numbers, $d(y)=d(y /|y|) \in C^{\infty}\left(E^{n} \backslash\{0\}\right)$, and $K_{1}$ is determined by $a, b, \sigma, c, d, n, N$, and $k \geq[n / 2]+1$.

Proof. In order to use the classic Schauder interior estimates, we have to fix the domains on which the coefficients of the equation are uniformly $C^{k+3, \alpha}$ continuous. Therefore, we choose the following fixed domains:

$$
\begin{aligned}
& \Omega_{0} \subset \Omega_{1} \subset \Omega_{2} \subset \Omega_{3} \subset B_{1 / \sigma}, \\
& \Omega_{0}=\{z|1 / 2<| z \mid<1\}, \\
& \Omega_{1}=\{z|(1+6 \sigma) / 16 \sigma<| z \mid<3 /(2+\sigma)\}, \\
& \left.\Omega_{2}=\{z \mid(1+2 \sigma) / 8 \sigma)<|z|<2 /(1+\sigma)\right\}, \\
& \Omega_{3}=\{z|1 / 4 \sigma<| z \mid<1 / \sigma\} .
\end{aligned}
$$

On $\Omega_{i}$, we scale our $v$ and $f$ again by setting

$$
v_{r}(z)=v(r z) \text { and } f_{r}(z)=f(r z)
$$

for $1 / 4 \sigma \leq|z| \leq 1 / \sigma$ and $r \leq \sigma$. Now our equation $\mathscr{L}_{0} v=f$ becomes

$$
a \sum_{i=1}^{n} \frac{\partial^{2} v_{r}}{\partial z_{i}^{2}}+b \sum_{i, j=1}^{n} \frac{z_{i} z_{j}}{|z|^{2}} \frac{\partial^{2} v_{r}}{\partial z_{i} \partial z_{j}}+c \frac{z_{i}}{|z|} \frac{\partial v_{r}}{\partial z_{i}}+\frac{d(z)}{|z|^{2}} v_{r}=r^{2} f(z) .
$$

We introduce the usual Hölder norms and Sobolev norms on $\Omega_{i}$ for $i=$ $0,1,2,3$ :

$$
\begin{gathered}
\|u\|_{C^{k, \alpha}\left(\Omega_{i}\right)}=\sum_{|j|=1}^{k} \sup _{z \in \Omega_{i}}\left\{\left|\partial^{j} u(z)\right|\right\}+\sup _{\substack{x \neq z \\
x, z \in \Omega_{i}}} \frac{\left|\partial^{k} u(x)-\partial^{k} u(z)\right|}{|x-z|^{\alpha}}, \\
\|u\|_{W^{k, 2}\left(\Omega_{i}\right)}=\left(\int \sum_{|j|=1}^{k}\left|\partial^{j} u\right|^{2} d z\right)^{1 / 2} .
\end{gathered}
$$

For $z \in \Omega_{3}, 1 / 4 \sigma<|z|<1 / \sigma$, the coefficients of $(2.9)_{r}$ are

$$
A_{i j}(z)=a \delta_{i j}+b \frac{z_{i} z_{j}}{|z|^{2}}, \quad c_{i}=\frac{c z_{i}}{|z|}, \frac{d(z)}{|z|^{2}} \text { for } i, j=1,2, \ldots, n,
$$

which are $C^{\infty}$ and independent of $r$. It is easy to see that $A:=\left(A_{i j}\right)$ is uniformly elliptic on $\Omega_{3}$. In what follows, we fix $r$ to be a constant number.

Our first step is to show that there is a constant $K_{2}$ which is independent of $v_{r}, f_{r}$, and $r$ such that

$$
\left\|v_{r}\right\|_{C^{k, \alpha}\left(\Omega_{0}\right)} \leq K_{2}\left\{\left\|v_{r}\right\|_{L^{2}\left(\Omega_{3}\right)}+r^{2}\left\|f_{r}\right\|_{C^{k-2, \alpha}\left(\Omega_{3}\right)}\right\} .
$$


For this purpose, we use the classical estimates (cf. [A, GT]) for equation (2.9) to get two constant numbers $K_{3}$ and $K_{4}$ such that

$$
\begin{gathered}
\left\|v_{r}\right\|_{W^{k, 2}\left(\Omega_{2}\right)} \leq K_{3}\left\{\left\|v_{r}\right\|_{L^{2}\left(\Omega_{3}\right)}+r^{2}\left\|f_{r}\right\|_{W^{k-2,2}\left(\Omega_{3}\right)}\right\}, \\
\left\|v_{r}\right\|_{C^{k, \alpha}\left(\Omega_{0}\right)} \leq K_{4}\left\{\left\|v_{r}\right\|_{C^{0}\left(\Omega_{1}\right)}+r^{2}\left\|f_{r}\right\|_{C^{k-2, \alpha}\left(\Omega_{1}\right)}\right\} .
\end{gathered}
$$

On the other hand, the Kondrakov-Sobolev Theorem (cf. [A]) implies that there is another constant $K_{5}$ such that

$$
\left\|v_{r}\right\|_{C^{0}\left(\Omega_{1}\right)} \leq K_{5}\left\|v_{r}\right\|_{W^{k, 2}\left(\Omega_{2}\right)} \text { for } k \geq[n / 2]+1,
$$

where $K_{3}, K_{4}$, and $K_{5}$ are independent of the choice of $v, f$, and $r$.

Using $(2.11)_{r}-(2.13)_{r}$, we have proved $(2.10)_{r}$ holds for all $r$, where $K_{2}$ is independent of $v_{r}, f_{r}$, and $r$.

By definition (2.6), one can show that

$$
\vdots v_{L_{N, \sigma}^{2}}=\sup _{0<r \leq \sigma}\left\{r^{-N}\left\|v_{r}\right\|_{L^{2}\left(\Omega_{3}\right)}\right\} .
$$

For the same reasons, we have

$$
\||v|\|_{k, \alpha ; N, \sigma}=\sup _{0 \leq r \leq \sigma}\left\{r^{-N}\left\|v_{r}\right\|_{C^{k, \alpha}\left(\Omega_{0}\right)}\right\},
$$

and we observe that

$$
\sup _{0 \leq r \leq \sigma}\left\{r^{2-N}\left\|f_{r}\right\|_{C^{k-2, \alpha}\left(\Omega_{3}\right)}\right\} \leq\||f|\|_{k-2, \alpha ; N-2}
$$

It follows form $(2.10)_{r}$ that

$$
r^{-N}\left\|v_{r}\right\|_{C^{k, a}\left(\Omega_{0}\right)} \leq K_{2}\left\{r^{-N}\left\|v_{r}\right\|_{L^{2}\left(\Omega_{3}\right)}+r^{2-N}\left\|f_{r}\right\|_{C^{k-2, \alpha}\left(\Omega_{3}\right)}\right\} .
$$

Therefore, (i)-(iii) and the supremum of (iv) give the lemma.

Now, we are ready to find the bounded linear inverse of $\mathscr{L}_{0}$.

Theorem 2.14. Suppose

$$
\mathscr{L}_{0}=a \Delta_{E^{n}}+b \frac{\partial^{2}}{\partial R^{2}}+\frac{c}{R} \frac{\partial}{\partial R}+\frac{d}{R^{2}}
$$

is a second order singular elliptic differential operator with $a>0$ and $b \geq 0$. Then, for any $1>\sigma>1 / 2, \varepsilon>1 / 2$, and $k \geq[n / 2]+1$, there exists a bounded linear right inverse $S$ of $\mathscr{L}_{0}$ such that

(i) $\mathscr{L}_{0} \circ S(f(y))=f(y)$ for all $|y| \leq \sigma$.

(ii) $S$ maps $C_{k-2, \alpha ; N} \cap C_{0, \alpha ; N-2+\varepsilon}$ to $C_{k, \alpha ; N}$ and

$$
\||S \circ f|\|_{k, \alpha ; N} \leq \widetilde{K}\left\{\||f|\|_{0, \alpha ; N-2+\varepsilon}+\||f|\|_{k-2, \alpha ; N-2}\right\},
$$

where $\widetilde{K}$ is a constant number which is independent of $v$ and $f$. 
Proof. We will seek $S_{0}$, the right inverse of $\mathscr{L}_{0}$, by a Fourier expansion as in [CHS] and use a cut-off function $\zeta$ to get $S=\zeta \circ S_{0}$. If $v=S_{0} f$, by estimates (2.8), we only care about the weighted $L^{2}$ norm of $v$. The rest of the proof is devoted to constructing $S_{0}$ and finding constant numbers $K_{14}$ and $1>\tau>\sigma$ such that

$$
\vdots v_{L_{N, \tau}^{2}} \leq K_{14}\||f|\|_{0, \alpha ; N-2+\varepsilon}, \quad \text { with } \varepsilon>1 / 2,
$$

where $K_{14}$ is independent of $v$ and $f$. It will take several pages to demonstrate $S_{0}$ and (2.15).

Suppose $\Delta_{E}$ and $\Delta^{*}$ are the intrinsic Laplacian operators on $E^{n}$ and the unit sphere $S^{n-1}$. For $y \in E^{n}$, let

$$
R=\left[\sum y_{i}^{2}\right]^{1 / 2}=|y|, \quad \theta=\frac{y}{|y|} \in S^{n-1} .
$$

Clearly, two Laplacian operators are related by

$$
\Delta_{E} v=\frac{\partial^{2} v}{\partial R^{2}}+\frac{n-1}{R} \frac{\partial v}{\partial R}+\frac{1}{R^{2}} \Delta^{*} v
$$

where $v=v(R, \theta)$ on the right side and $\Delta^{*} v$ stands for $\Delta_{S^{n-1}}[v(R, \circ)]$.

On the unit sphere $S^{n-1}$, the eigenvalues of the linear operator $\left(-a \Delta^{*}-d\right)$ may be written as $\lambda_{0} \leq \lambda_{1} \leq \lambda_{2} \leq \cdots$, and the corresponding eigenfunctions as $\varphi_{1}, \varphi_{2}, \varphi_{3}, \ldots$, chosen to form an orthonormal basis for $L^{2}\left(S^{n-1}\right)$. Each such eigenfunction is the harmonic polynomial of $E^{n}$ and

(see $[\mathrm{BGE}])$.

$$
\lambda_{i} \in\{a k(k+n-2)+d\}_{0 \leq k \leq+\infty}
$$

The shapes of Euclidean spheres permit a further separation of variables for the solutions of $\mathscr{L}_{0} v=f, f \in C_{k-2, \alpha ; N}$. Obviously,

$$
v(R, \theta)=\sum_{m=0}^{+\infty} \beta_{m}(R) \circ \varphi_{m}(\theta)
$$

is a formal solution of $\mathscr{L}_{0} v=f$ if and only if for all $m=0,1,2, \ldots$

$$
(a+b) \beta_{m}^{\prime \prime}(R)+\frac{[a(n-1)+c] \beta_{m}^{\prime}(R)}{R}-\frac{\lambda_{m}}{R^{2}} \beta_{m}(R)=f_{m}(R)
$$

holds, where $f_{m}=\int_{S^{n-1}} f(R, \theta) \varphi_{m}(\theta) d \theta$ and $d \theta$ is the standard area element on the unit sphere.

Let

$$
\begin{aligned}
& \gamma_{m}=\frac{-a(n-2)+b-c+\sqrt{(n a-2 a-b+c)^{2}+4 \lambda_{m}(a+b)}}{2(a+b)}, \\
& \eta_{m}=\frac{-a(n-2)+b-c-\sqrt{(n a-2 a-b+c)^{2}+4 \lambda_{m}(a+b)}}{2(a+b)} .
\end{aligned}
$$


There are solutions of $(2.17)_{m}$

$$
\begin{aligned}
\beta_{m}(R)=\operatorname{Re}\left\{R^{\gamma_{m}}\right. & \int_{c_{m}}^{R} \frac{f_{m}(s) \circ(s)^{-\gamma_{m}+1}}{(a+b)\left(\gamma_{m}-\eta_{m}\right)} d s \\
& \left.-R^{\eta_{m}} \int_{d_{m}}^{R} \frac{f_{m}(s) \circ(s)^{-\eta_{m}+1}}{(a+b)\left(\gamma_{m}-\eta_{m}\right)} d s\right\}
\end{aligned}
$$

for $m=0,1,2, \ldots$, where $c_{m}$ and $d_{m}$ are constants to be chosen later. In the case $\left(\gamma_{m_{0}}-\eta_{m_{0}}\right)=0$ for some $m_{0}$, we let

$$
\begin{aligned}
\beta_{m_{0}}(R)=\frac{-R^{\gamma_{m_{0}}}}{(a+b)}\left\{\int_{c_{m_{0}}}^{R} f_{m_{0}}(s) s^{-\gamma_{m_{0}}+1} \circ(\log s) d s\right. & \\
& \left.-\log R \int_{d_{m_{0}}}^{R} f_{m_{0}}(s) s^{-\gamma_{m_{0}}+1} d s\right\} .
\end{aligned}
$$

We take two integers $J_{1}$ and $J_{2}$ such that

Now, the expression

$$
\operatorname{Re} \gamma_{J_{1}} \leq N<\operatorname{Re} \gamma_{J_{1}+1}, \quad \operatorname{Re} \eta_{J_{2}+1} \leq N-1<\operatorname{Re} \eta_{J_{2}} .
$$

$$
v(R, \theta)=\sum_{m=0}^{+\infty} \beta_{m}(R) \circ \varphi_{m}(\theta)
$$

makes sense and gives a weak solution $\mathscr{L}_{0} v=f$, if we take

$$
\begin{aligned}
& c_{m}=0 \text { for } m \leq J_{1}, \quad c_{m}=1 \text { for } m \geq J_{1}+1 \text {, } \\
& d_{m}=1 \text { for } m \leq J_{2}, \quad d_{m}=0 \text { for } m \geq J_{2}+1 \text {, }
\end{aligned}
$$

since we have the following $L^{2}$ estimates: if $m>\max \left\{J_{1}, J_{2}\right\}, m \neq m_{0}$, and $R<1$, then

$$
\begin{aligned}
\beta_{m}^{2}(R)=\left[R^{\gamma_{m}} \int_{1}^{R} \frac{f_{m}(s) \circ(s)^{-\gamma_{m}+1}}{(a+b)\left(\gamma_{m}-\eta_{m}\right)} d s-R^{\eta_{m}} \int_{0}^{R} \frac{f_{m}(s) \circ(s)^{-\eta_{m}+1}}{(a+b)\left(\gamma_{m}-\eta_{m}\right)} d s\right]^{2} \\
\leq \frac{2}{\left[(a+b)\left(\gamma_{m}-\eta_{m}\right)\right]^{2}}\left\{\left|\left[\int_{1}^{R} f_{m}(s) \circ(s)^{(-N+2-1 / 2)-\gamma_{m}+N-1 / 2} d s\right]^{2}\right| R^{2 \gamma_{m}}\right. \\
\left.+\left[R^{2 \eta_{m}} \int_{0}^{R} f_{m}^{2}(s) \circ(s)^{3-2 N} d s \int_{0}^{R}(s)^{-1-2 \eta_{m}+2 N} d s\right]\right\} \\
\leq \frac{2 R^{2 N}}{\left[(a+b)\left(\gamma_{m}-\eta_{m}\right)\right]^{2}}\left|\int_{0}^{1} f_{m}^{2}(s) \circ(s)^{3-2 N} d s\right|\left[\frac{2}{\left|N-\gamma_{m}\right|}+\frac{1}{\left|N-\eta_{m}\right|}\right] .
\end{aligned}
$$

For all other $m \neq m_{0}, \eta_{m} \neq N \neq \gamma_{m}$, we also have

$$
\begin{aligned}
\beta_{m}^{2}(R) \leq & \frac{2 R^{2 N}}{\left[(a+b)\left(\gamma_{m}-\eta_{m}\right)\right]^{2}} \\
& \times\left|\int_{0}^{1} f_{m}^{2}(s) \circ(s)^{3-2 N} d s\right|\left[\frac{2}{\left|N-\gamma_{m}\right|}+\frac{1}{\left|N-\eta_{m}\right|}\right] .
\end{aligned}
$$


In the remaining cases, one can verify that

$$
\left|\beta_{m}(R)\right| \leq K_{7} \max _{y \in B_{1}}\left\{\frac{f(y)}{|y|^{N-2+\varepsilon}}\right\} R^{N}, \quad \text { when } \varepsilon>\frac{1}{2},
$$

where $K$ is a constant which is independent of $f$. Using Parseval's identity and the orthogonality of the $\varphi_{m}$ 's we readily verify that

$$
\begin{aligned}
\int_{S^{n-1}} v^{2}(R, \theta) d \theta & =\sum_{m=0}^{+\infty} \beta_{m}^{2}(R, \theta) \\
& \leq K_{8}\left\{\sum_{m=0}^{+\infty} R^{2 N} \int_{0}^{1} \frac{f_{m}^{2}(s)}{s^{2 N-3}} d s+R^{2 N}\left\|\frac{f()}{||^{N-2+\varepsilon}}\right\|_{C^{0}}^{2}\right\} .
\end{aligned}
$$

In other words, our solution $v$ satisfies

$$
\begin{aligned}
|v|_{L_{N, \tau}^{2}} & =\sup _{0 \leq r \leq \tau}\left\{r^{-N}\left[\int_{1 / 4 \tau}^{1 / \tau} \int_{S^{n-1}} t^{n-1} v^{2}(r t, \theta) d \theta d t\right]^{1 / 2}\right\} \\
& \leq K_{8}^{1 / 2}\left(\int_{1 / 4 \tau}^{1 / \tau} t^{n-1+2 N} d t\right)^{1 / 2}\left\{\int_{0}^{1} \frac{1}{s^{2 N-3}} \int_{S^{n-1}} f^{2}(s, \theta) d \theta\right. \\
& \left.+\left\|\frac{f()}{\mid l^{N-2+\varepsilon}}\right\|_{C^{0}}^{2} d s\right\}^{1 / 2} \\
& \leq K_{14}\||f|\|_{0,0 ; N-2+\varepsilon}, \quad \text { with } \varepsilon>1 / 2 .
\end{aligned}
$$

Thus, we have constructed $S_{0}$ and (2.15) was verified.

In order to use the interior estimates, we introduce a cut-off function $\zeta$ such that

Finally, we define

$$
\zeta(y)= \begin{cases}1 & \text { for }|y| \leq \sigma, \\ 0 & \text { for }|y| \geq \tau, \text { with } 0<\sigma<\tau<1 .\end{cases}
$$

$$
\tilde{v}=S \circ f=\zeta() v()=\zeta S_{0} \circ f .
$$

Using Lemma 2.7 and (2.15), we get

$$
\begin{aligned}
\||S \circ f|\|_{k, \alpha ; N} & =\||\tilde{v}|\|_{k, \alpha ; N, \tau} \leq K_{g}\||v|\|_{k, \alpha ; N, \tau} \\
& \leq K_{g} K_{1}\left\{2\||f|\|_{k-2, \alpha ; N-2}+K_{14}\||f|\|_{0, \alpha ; N-2+\varepsilon}\right\} .
\end{aligned}
$$

This completes the proof of Theorem 2.14.

Corollary 2.14. Let $\mathscr{L}_{0}$ and $1>\sigma>1 / 2$ be as in Theorem 2.14 , and let $Z_{1}=\bigcap_{k, N}^{\infty} C_{k, \alpha ; N}$. Then there is a linear operator $\mathscr{S}$ on $Z_{1}$ such that:

(i) $\mathscr{L}_{0}(\mathscr{S} f)(y)=f(y)$ for all $|y| \leq \sigma$.

(ii) If $f^{t}(y)=f(t y), 0<t \leq 1$ and $|y| \leq \sigma$, then $\left(\mathscr{S} f^{t}\right)(y)=t^{-2}(\mathscr{S} f)(t y)$.

(iii) For any $k, \alpha, N$, and $\varepsilon>1 / 2$, there is a constant number $\widetilde{K}$ such that

$$
\||\mathscr{S} \circ f|\|_{k, \alpha ; N} \leq \widetilde{K}\left\{\||f|\|_{0, \alpha ; N-2+\varepsilon}+\||f|\|_{k-2, \alpha ; N-2}\right\} \text {. }
$$


Proof. We use the same notation and methods as in the proof of Theorem 2.14 with some modifications. First, we want to define $\mathscr{S}$ and verify (ii). Let $c_{m}=d_{m}=0$ for all $m=0,1, \ldots$, and let $\varphi_{m}, \gamma_{m}, \eta_{m}, \zeta, R, \theta$, and $y=R \theta$ be as in the proof of Theorem 2.14. Setting

$$
f_{m}=\int_{S^{n-1}} f(R, \theta) \varphi_{m}(\theta) d \theta,
$$

we also define

$$
\begin{aligned}
\beta_{m}(f, R)=\operatorname{Re}\left\{R^{\gamma_{m}}\right. & \int_{0}^{R} \frac{f_{m}(s) \circ(s)^{-\gamma_{m}+1}}{(a+b)\left(\gamma_{m}-\eta_{m}\right)} d s \\
& \left.-R^{\eta_{m}} \int_{0}^{R} \frac{f_{m}(s) \circ(s)^{-\eta_{m}+1}}{(a+b)\left(\gamma_{m}-\eta_{m}\right)} d s\right\} .
\end{aligned}
$$

If $\gamma_{m_{0}}=\eta_{m_{0}}$, we let

$$
\begin{aligned}
\beta_{m_{0}}(f, R)=\frac{-R^{\gamma_{m_{0}}}}{(a+b)}\left\{\int_{0}^{R}\right. & f_{m_{0}}(s) s^{-\gamma_{m_{0}}+1} \circ(\log s) d s \\
& \left.\quad-\log R \int_{0}^{R} f_{m_{0}}(s) s^{-\gamma_{m_{0}}+1} d s\right\} .
\end{aligned}
$$

When $f^{t}(y)=f(t y), f \in Z_{1}$, and $\tilde{s}=t s$, one can prove

$$
\begin{aligned}
& \beta_{m}\left(f^{t}, R\right)= \operatorname{Re}\left\{R^{\gamma_{m}} \int_{0}^{R} \frac{f_{m}^{t}(s) \circ(s)^{-\gamma_{m}+1}}{(a+b)\left(\gamma_{m}-\eta_{m}\right)} d s\right. \\
&\left.-R^{\eta_{m}} \int_{0}^{R} \frac{f_{m}^{t}(s) \circ(s)^{-\eta_{m}+1}}{(a+b)\left(\gamma_{m}-\eta_{m}\right)} d s\right\} \\
&=\frac{1}{t^{2}} \operatorname{Re}\left\{(t R)^{\gamma_{m}} \int_{0}^{t R} \frac{f_{m}(\tilde{s}) \circ(\tilde{s})^{-\gamma_{m}+1}}{(a+b)\left(\gamma_{m}-\eta_{m}\right)} d \tilde{s}\right. \\
&\left.\quad-(t R)^{\eta_{m}} \int_{0}^{R} \frac{f_{m}(\tilde{s}) \circ(\tilde{s})^{-\eta_{m}+1}}{(a+b)\left(\gamma_{m}-\eta_{m}\right)} d \tilde{s}\right\} \\
&=t^{-2} \beta_{m}(f, t R) .
\end{aligned}
$$

Similarly, one can verify that $(2.20)_{m}$ holds when $m=m_{0}$. Now, we define

$$
(\mathscr{S} f)_{(R, \theta)}=\zeta(R) \sum_{m=0}^{\infty} \beta_{m}(f, R) \varphi_{m}(\theta) .
$$

When $0<t<1$ and $R=|y|<\sigma$, we have $\zeta(t y)=1=\zeta(y)$, and (ii) follows from $(2.20)_{m}, m=0,1, \ldots$.

The same argument as in proof of Theorem 2.14 shows that for any given $k, \alpha$, and $N$ there is a constant number $\widetilde{K}$ so that (i) and (iii) still hold. 


\section{THE PROOF OF THE MAIN THEOREM}

In this section, we will prove the main theorem under the following extra assumption:

$$
\left\{\operatorname{det}\left(g_{i j}^{0}(x)\right)-1\right\} \in Z_{\tau} \quad \text { for some } \tau>0 .
$$

(It was pointed out by Professor Jerry Kazdan to the author that any other Riemannian metric is locally conformal to one of those metrics mentioned in assumption (A).)

According to Theorem A (see $\S 1$ ), we only have to solve the equation

$$
V\left(x, \partial w, \partial^{2} w\right)=-\frac{\partial_{r}\left(\log \sqrt{\operatorname{det}\left(g_{i j}^{0}\right)}\right)}{r}
$$

under assumption (A). The rest of this section is devoted to solving equation $(* *)$ in the case that $(\mathrm{A})$ holds.

In fact, we shall consider more general singular elliptic equations. Let $\left\{x_{i}\right\}$ be $g^{0}$-geodesic normal coordinates about $p$, and

$$
\begin{aligned}
& r(x)=d_{g^{0}}(x, p), \quad \Delta_{E^{n}}=\sum \frac{\partial^{2}}{\partial x_{i}^{2}}, \\
& \mathscr{L}_{0}=a \Delta_{E^{n}}+b \frac{\partial^{2}}{\partial r^{2}}+\frac{c}{r} \frac{\partial}{\partial r}+\frac{d}{r^{2}} \quad \text { with } a>0, b \geq 0, \\
& V\left(x, w, \partial_{x} w, \partial_{x}^{2} w\right) \\
& =\mathscr{L}_{0} w+\sum Q_{i j}(x, w, \partial w) \partial_{x_{i}} \partial_{x_{j}} w+G\left(x, w, \partial_{x} w\right),
\end{aligned}
$$

with the conditions

$$
\begin{aligned}
Q_{i j}(x, \zeta, \eta) & =\sum x_{k} Q_{i j, k}(x, \zeta, \eta), \\
G(x, \zeta, \eta) & =\sum \frac{x_{i} x_{j}}{|x|^{2}} G_{i j}(x, \zeta, \eta),
\end{aligned}
$$

where $Q_{i j, k}$ and $G_{i j}$ are $C^{\infty}$ smooth functions with respect to $x, \zeta, \eta$. When $|x| \leq \rho_{0}$, we also require that $G_{i j}(x, 0,0)=0$.

Using the fact $r \partial w / \partial r=\sum x_{i} \partial w / \partial x_{i}$, one can verify that $V$ given in (1.14) satisfies conditions (i) and (ii).

We will prove

Theorem B. Suppose $V$ is a second order quasilinear elliptic operator on a Riemannian manifold $\left(M, g^{0}\right)$ with an isolated singularity $p$ with conditions (i) and (ii) as above. Then there is a $\tau>0$ such that

$$
V\left(x, w, \partial_{x} w, \partial_{x}^{2} w\right)=f(x)
$$

has a solution $w \in C_{k, \alpha ; N, \rho}$ for any $\rho<\tau, \varepsilon>1 / 2, k \geq[n / 2]+1$, and $f \in C_{k-2, \alpha ; N} \cap C_{0, \alpha ; N-2+\varepsilon}$. 
Corollary B. Let $V$ and $\mathscr{L}_{0}$ be as above, and let $f \in Z_{\tau}$. Then $(* * *)$ has a solution $w \in Z_{\rho}$ for sufficiently small $\rho<\tau$.

Proof of the Theorem B. The idea of the proof will be to use the Schauder fixed point theorem. To set the stage for the fixed point theorem, we need to fix the domains of the functions involved so that we can deal with some fixed Banach spaces. There is no loss of generality in assuming the injective radius of $g^{0}$ at $p$ is greater than one. Thus, we scale our problem the same way Dennis DeTurck did in [D]. If $v$ is a function defined on the metric ball $B_{1}$, we set

$$
\Phi(\rho, v)=V\left(\rho y, \rho^{2} v, \rho \partial_{y} v, \partial_{y}^{2} v\right)-f(\rho y) .
$$

In this case, $\Phi(\rho, v)$ is a function defined on $B$. Since $f(0)=0$ and $G(0,0,0)=0=Q_{i j}(0,0,0)$, we see that $\Phi(0, v)=\mathscr{L}_{0} v$.

Let $S$ be as in Theorem 2.14. Then $S$ is a bounded linear right inverse of $\mathscr{L}_{0}$. Define

$$
\begin{aligned}
T_{\rho}(v) & =\Phi(0, v)-\Phi(\rho, v) \\
& =\mathscr{L}_{0} v-V\left(\rho y, \rho^{2} v, \rho \partial_{y} v, \partial_{y}^{2} v\right)+f(\rho y) \\
& =f(\rho y)-\sum Q_{i j}\left(\rho y, \rho^{2} v, \rho \partial_{y}^{2} v\right) \frac{\partial^{2} v}{\partial y_{i} \partial y_{j}}-G\left(\rho y, \rho^{2} v, \rho \partial_{y}^{2} v\right)
\end{aligned}
$$

$$
F_{\rho}(v)=S\left[T_{\rho}(v)\right]
$$

Now, if $F_{\rho}$ admits a fixed point $v$ in $C_{k, \alpha ; N}$, and $\rho>0$, then

$$
V\left(\rho y, \rho^{2} v, \rho \partial_{y} v, \partial_{y}^{2} v\right)-f(\rho y)=0 \text { for }|y| \leq 1 / 2 .
$$

Setting $w(x)=\rho^{2} v(x / \rho)$ gives a solution of $(* * *)$ for $|x| \leq \rho / 2$.

For this purpose, we want to verify that $F_{\rho}$ is a contracting map on some convex bounded set $\mathfrak{H}_{K_{0}}=\left\{v\left|\||v|\|_{k, \alpha ; N} \leq K_{0}\right\}\right.$. For any pair of functions $v$, $\tilde{v} \in \mathfrak{H}_{K_{0}}$, condition (ii) and the definition of $\|||\|_{k, \alpha ; N}$ tell us that

$$
\begin{aligned}
& \left\|\left|T_{\rho}(\tilde{v})-T_{\rho}(v)\right|\right\|_{k-2, \alpha ; N-2}+\left\|\left|T_{\rho}(\tilde{v})-T_{\rho}(v)\right|\right\|_{0, \alpha ; N-2+\varepsilon} \\
& \quad \leq K_{3} \rho\||\tilde{v}-v|\|_{k, \alpha ; N},
\end{aligned}
$$

where $K_{3}$ is a constant number which depends on $k, \alpha, N$, and $K_{0}$. On the other hand, by Theorem 2.14 we know that there is a constant $\widetilde{K}$ so that

$$
\||S \circ \tilde{f}|\|_{k, \alpha ; N} \leq \tilde{K}\left\{\||\tilde{f}|\|_{0, \alpha ; N-2+\varepsilon}+\||\tilde{f}|\|_{k-2, \alpha ; N-2}\right\}
$$

for any $\tilde{f} \in C_{k-2, \alpha ; N} \cap C_{0, \alpha ; N-2+\varepsilon}$.

Now setting $T_{\rho}(\tilde{v})-T_{\rho}(v)=\tilde{f}$ in (3.3) yields that, if $v, \tilde{v} \in \mathfrak{H}_{K_{0}}$, then

$$
\left\|\left|F_{\rho}(v)-F_{\rho}(\tilde{v})\right|\right\|_{k, \alpha ; N} \leq \tilde{K} K_{3} \rho\||\tilde{v}-v|\|_{k, \alpha ; N} .
$$


The fixed point of $v$ of $F_{\rho}$ can be constructed as a limit of a sequence of functions. Let

$$
\begin{aligned}
& u_{0, \rho}=0, \quad u_{1, \rho}=F_{\rho}(0)=S \cdot f(\rho \cdot), \\
& u_{i, \rho}=F_{\rho}\left(u_{i-1}, \rho\right) .
\end{aligned}
$$

Now we let $K_{0}=8\||S f|\|_{k, \alpha ; N}$, and choose $\rho_{k, N}=1 / 4\left(\tilde{K} K_{3}+K_{0}\right)$. One can show that, if $\tau=\rho_{k, N}$ and $\rho<\tau$, then $\left\{u_{i, \rho}\right\} \subset \mathfrak{H}_{K_{0}}$ and

$$
\left\|\left|u_{i, \rho}-u_{i-1, \rho}\right|\right\|_{k, \alpha ; N} \leq \frac{K_{0}}{2^{i-1}} \text {. }
$$

Hence, $u_{\infty, \rho}=\lim _{i \rightarrow \infty} u_{i, \rho}$ is the desired fixed point of $F_{\rho}$.

Proof of Corollary B. We make a few changes in the proof of Theorem B and use the same notation unless specified otherwise. Recall that

$$
\Phi(\rho, v)=V\left(\rho y, \rho^{2} v, \rho \partial_{y} v, \partial_{y}^{2} v\right)-f(\rho y),
$$

and let $\mathscr{S}$ be given as in Corollary 2.14. We also define

$$
\begin{aligned}
\tilde{F}_{\rho}(v) & =\mathscr{S}[\Phi(0, v)-\Phi(\rho, v)] \\
& =\mathscr{S}\left[\mathscr{L}_{0} v-V\left(\rho y, \rho^{2} v, \rho \partial_{y} v, \partial_{y}^{2}\right)+f(\rho y)\right], \\
\tilde{u}_{0, \rho} & =0, \quad \tilde{u}_{1, \rho}=\widetilde{F}_{\rho}(0)=\mathscr{S} \cdot f(\rho \cdot), \\
\tilde{u}_{i, \rho} & =\widetilde{F}_{\rho}\left(u_{i-1}, \rho\right), \\
\tilde{u}_{\infty, \rho} & =\lim _{i \rightarrow \infty} \tilde{u}_{i, \rho} .
\end{aligned}
$$

Now, for any $k, \alpha, N$, using the same argument as in the proof of Theorem B, we have a constant $\rho_{k, N}$ so that, when $\rho<\rho_{k, N}$

$$
\left\|\left|\tilde{u}_{i, \rho}-\tilde{u}_{i-1, \rho}\right|\right\|_{k, \alpha ; N} \leq \frac{K_{0}}{2^{i-1}},
$$

where $K_{0}=8\||\mathscr{S} f|\|_{k, \alpha ; N}$. Hence, $\tilde{u}_{\infty, \rho} \in C_{k, \alpha ; N}$.

If we fix $\tau=\frac{1}{2} \rho_{2 n, 1 / 2 ; 4 n}, n=\operatorname{dim} M$, and let

$$
v_{0}=\tilde{u}_{\infty, \tau}, \quad w_{0}(x)=\tau^{2} v\left(\frac{x}{\tau}\right),
$$

then we shall show that $w_{0}$ is the desired $C^{\infty}$ smooth solution of $(* * *)$.

In fact, by (3.9), one can show that $w_{0}$ is a strong solution of $(* * *)$ for $0<|x| \leq \tau / 2$. Since $V$ is elliptic on $B_{1}-\{0\}$ and the coefficient of $V$ is $C^{\infty}$ at $B_{1}-\{0\}$, the standard elliptic theory tells us that $w_{0}$ is $C^{\infty}$ on $B_{1 / 2}-\{0\}$ when $f$ is $C^{\infty}$. Thus, we proved that $w_{0} \in C^{\infty}\left(B_{1 / 2}-\{0\}\right)$.

For the rest of the proof we show $v_{0}$ is $C^{\infty}$ at $y=0$ and $v_{0} \in Z_{1}$. We will prove this fact indirectly. The crucial step is to verify

Claim. If $\tilde{u}_{\infty, \rho}$ is given by (3.8), then

$$
\tilde{u}_{\infty, \rho}(t y)=t^{2} \tilde{u}_{\infty, t \rho}(t y) \text { for }|y| \leq 1 / 2, t \leq 1 .
$$


Assuming the claim, we see that $v_{0}(t y)=t^{2} \tilde{u}_{\infty, t \tau}(t y)$. Now, for any given $k, \alpha$, and $N$, choosing $t$ with $t \tau<\rho_{k, N}$ and using (3.9) ${ }_{i}-(3.11)$, we conclude $v_{0} \in C_{k, \alpha ; N}$. Therefore, it follows that $v_{0} \in Z_{1 / 2}$ and Corollary B holds.

In order to prove the claim, it suffices to verify

$$
\tilde{u}_{i, \rho}(t y)=t^{2} \tilde{u}_{i, t \rho}(t y) \text { for }|y| \leq 1 / 2, t \leq 1,
$$

for each $i$.

When $i=1$, if we let $v_{t}(y)=v(t y)$ and $h(y)=f(\rho y)$, then

$$
\begin{gathered}
\Phi\left(t \rho, v_{t}\right)_{(y)}=V\left(\rho t y, t^{2} \rho^{2} v_{t}, t \rho \partial_{y} v_{t}, \partial_{y}^{2} v_{t}\right)-f(t \rho y) \\
=\left.\Phi\left(\rho, t^{2} v\right)\right|_{t y}, \\
\left.T_{t \rho}\left(v_{t}\right)\right|_{y}=\left.T_{\rho}\left(t^{2} v\right)\right|_{t y} .
\end{gathered}
$$

Let $\mathscr{S}$ be as in Corollary 2.14. If we set $h_{t}(y)=h(t y)=f(t \rho y)$, it follows from Corollary 2.14(ii) that

$$
\left.\mathscr{S}(h)\right|_{t y}=\left.t^{2} \mathscr{S}\left(h_{t}\right)\right|_{y} \quad \text { if }|y| \leq 1 / 2 \text { and } t \leq 1 .
$$

Therefore, setting $v=t^{-2} \mathscr{S}(h)$ in (3.12) and using (3.13), we find that

$$
\begin{aligned}
\left.T_{t \rho}\left(\mathscr{S}\left(h_{t}\right)\right)\right|_{y} & =\left.T_{t \rho}\left[\left(t^{-2} \mathscr{S} h\right)_{(t \cdot)}\right]\right|_{y} \\
& =\left.T_{\rho}[\mathscr{S} h]\right|_{t y} \quad \text { when }|y| \leq 1 / 2, t \leq 1, \\
\tilde{u}_{1, \rho}(t y) & =\left.(\mathscr{S} h)\right|_{t y}=\left.t^{2}\left(\mathscr{S} h_{t}\right)\right|_{y} \\
& =\left.t^{2}[\mathscr{S} f(t \rho \cdot)]\right|_{y}=t^{2} \tilde{u}_{1, t \rho}(y) .
\end{aligned}
$$

When $i \geq 2$, suppose

$$
\left.\left[\left(T_{t \rho} \mathscr{S}\right)^{i-1}\left(h_{t}\right)\right]\right|_{y}=\left.\left[\left(T_{\rho} \mathscr{S}\right)^{i-1} h\right]\right|_{t y}
$$

holds for $i-1$. Then one can verify that

$$
\begin{aligned}
{\left.\left[\left(T_{t \rho} \mathscr{S}\right)^{(i)}\left(h_{t}\right)\right]\right|_{y} } & =\left.\left\{\left(T_{t \rho} \mathscr{S}\right)\left[\left(T_{t \rho} \mathscr{S}\right)^{i-1} h_{t}\right]\right\}\right|_{y} \\
& =\left.\left\{\left(T_{t \rho} \mathscr{S}\right)\left[\left(T_{\rho} \mathscr{S}\right)^{i-1} h\right]_{\left(t^{*}\right)}\right\}\right|_{y} \\
& =\left.\left\{\left(T_{\rho} \mathscr{S}\right)\left[\left(T_{\rho} \mathscr{S}\right)^{i-1} h\right]\right\}\right|_{t y} \\
& =\left.\left[\left(T_{\rho} \mathscr{S}\right)^{(i)}(h)\right]\right|_{t y} .
\end{aligned}
$$

Hence, one concludes that

$$
\begin{aligned}
\tilde{u}_{i, \rho}(t y) & =\left.\left\{\mathscr{S}\left[\left(T_{\rho} \mathscr{S}\right)^{i-1} f(\rho \cdot)\right]\right\}\right|_{t y} \\
& \left.=\left.t^{2}\left\{\mathscr{S}\left[\left(T_{\rho} \mathscr{S}\right)^{i-1} f(\rho \cdot)\right]_{t}\right\}\right|_{y} \quad(\text { by })(3.13)\right) \\
& =\left.t^{2}\left\{\mathscr{S}\left[\left(T_{t \rho} \mathscr{S}\right)^{i-1} f(t \rho \cdot)\right]\right\}\right|_{y} \quad\left(\text { by }(3.14)_{i-1}\right) \\
& =\left.t^{2}\left\{\left(\widetilde{F}_{t \rho}\right)^{(i)}(0)\right\}\right|_{y}=t^{2} \tilde{u}_{i, t \rho}(y) .
\end{aligned}
$$

This completes the proof of the claim and corollary. 
In order to prove the main theorem by using Corollary B, we need an "infinite order version" of Lee and Parker's theorem. The author owes this remark to Professor J. Kazdan.

Theorem C. Suppose $\left(M, g^{0}\right)$ is a Riemannian metric around $p$. Then there exists a pointwise conformal metric $\tilde{g}=e^{2 h} g^{0}$ such that in $\tilde{g}$-normal coordinates $\left\{\tilde{x}_{1}, \tilde{x}_{2}, \ldots, \tilde{x}_{n}\right\}$ around $p$,

$$
\left.(\text { A.1 })_{\beta} \quad \frac{\partial^{\beta}}{\partial \tilde{x}^{\beta}}\left[\operatorname{det}\left(\tilde{g}_{i j}(\tilde{x})\right)-1\right]\right|_{\tilde{x}=0}=0 \text { for all }|\beta|=0,1,2, \ldots
$$

The following simple proof was suggested by the referee.

Proof of Theorem C. The theorem above will immediately follow from the result in [LP] and Borel's theorem, which says: for any power series about 0 in $E^{n}$, there exists a smooth $C^{\infty}$ real-valued function whose Taylor series expansion at 0 is this given series (cf. [GG, p. 98]).

To see this, starting with a metric $g_{0}$, we seek a metric $\tilde{g}=e^{2 h} g_{0}$ such that $\operatorname{det}\left(g_{i j}\right)-1$ vanishes to infinite order at $p$ in $\tilde{g}$-normal coordinates. This is equivalent to the coordinates free condition $\exp _{\tilde{g}}^{*}\left(d V_{\tilde{g}}\right)=d x$ to infinite order, where $d V_{\tilde{g}}$ is the volume element of $\tilde{g}$ and $d x$ is the Euclidean volume element on the tangent space at $p$. In Lemma 5.3 of [LP] it is shown that, for any $m$, there exists a metric $g_{m}=e^{2 h_{m}} g_{0}$ which satisfies $\exp _{g_{m}}^{*}\left(d V_{g_{m}}\right)=$ $\left[1+O\left(\|x\|^{m}\right)\right] d x$ and, as long as $h_{m}$ and its first derivatives vanish at $p$, the Taylor series of $h_{m}$ is uniquely determined up through order $m+2$ by this condition. Thus the full Taylor series of $h$ is uniquely determined by the condition that $\exp _{\tilde{g}}^{*}\left(d V_{\tilde{g}}\right)=\left[1+O\left(\|x\|^{m}\right)\right] d x$ for every $m$. Borel's theorem then guarantees that there exists a smooth function $h$ with this Taylor series.

Proof of the main theorem. Case 1. If $g^{0}$ is $C^{\infty}$ smooth, using Theorem C above we may assume that $\left\{\operatorname{det}\left(g_{i j}^{0}(x)\right)-1\right\} \in Z_{1}$ with respect to $g^{0}$-normal coordinates $\left\{x_{i}\right\}$ at $p$. Now the main theorem follows form Theorem $A$ and Corollary B.

Case 2. When $g^{0}$ is a $C^{k, \alpha}$ metric, according to Theorem 5.1 in [LP], we may assume that $\left\{\operatorname{det}\left(g_{i j}^{0}\right)-1\right\} \in C_{k, \alpha ; N}$. Therefore, one can prove the main theorem in this case by using Theorems $\mathbf{A}$ and $\mathbf{B}$.

\section{ACKNOWLEDGMENT}

The author would like to express his deep gratitude to Professor Jerry L. Kazdan who referred Borel's theorem and other penetrating insights to him which led the author to prove Theorem $\mathrm{C}$ and the main theorem in this paper. Many thanks are also due to Professor Fanghua Lin for bringing Caffarelli, Hardt, and Simon's paper to the author's attention. The author is grateful to Mr. Ziren Jin for his careful proofreading and many valuable suggestions. He 
would also like to thank Professors Chris Croke, Dennis DeTurck, Weiyue Ding, and Mr. Georgi Kamberov for their stimulating conversations and interest in his work.

Finally, we want to thank the referee for suggesting a simple proof of Theorem $C$ and pointing out several misprints and minor errors in an early version of this paper.

\section{REFERENCES}

[A] T. Aubin, Nonlinear analysis on manifolds. Monge-Ampere equations, Springer-Verlag, 1982.

[BE] A. Besse, Einstein manifolds, Springer-Verlag, 1986.

[BGE] M. Berger, P. Gauduchon, and E. Mazet, Le spectre d'une variete Riemannienne, SpringerVerlag, 1971.

[CHS] L. Caffarelli, R. Hardt, and L. Simon, Minimal surfaces and isolated singularities, Manuscripta Math. 48 (1984), 1-18.

[D] D. DeTurck, Existence of metrics with prescribed Ricci curvature, Invent. Math. 65 (1985), 197-207.

[DK] D. DeTurck and J. Kazdan, Some regularity theorem in Riemannian geometry, Ann. Sci. École Norm. Sup. (4) 14 (1981), 249-260.

[EH] J. Eschenberg and E. Heintze, An elementary proof of Cheeger-Gromoll splitting theorem, Global Analysis Geometry 2 (1984), 141-167.

[GT] D. Gilbarg and N. S. Trudinger, Elliptic partial differential equation of second order, Springer-Verlag, 1983.

[GG] M. Golubitsky and V. Guillemen, Stable mapping and their singularities, Springer-Verlag, 1973.

[GV] A. Gray and L. Vanhecke, Riemannian geometry and its determined by the volume of small geodesic balls, Acta Math. 142 (1979), 158-198.

[LP] J. Lee and T. Parker, Yamabe problem, Bull. Amer. Math. Soc. (N.S.) 17 (1987), 37-91.

[M] J. Milnor, Morse theory, Princeton Univ. Press, Princeton, N.J., 1963.

Department of Mathematics, University of Pennsylvania, Philadelphia, PennsylvaNIA 19104

Current address: Department of Mathematics, White Hall, Cornell University, Ithaca, New York 14853 